\title{
Microanalysis Software: Properties and Requirements
}

\author{
U. Rossek, R. Terborg, M. Falke, A. Kaeppel, M. Rohde \\ Bruker Nano GmbH, Schwarzschildstr. 12, 12489 Berlin, Germany
}

Energy dispersive X-ray spectrometry (EDX) on scanning electron microscopes (SEM) and similar instruments has become a standard technique for a wide range of applications. Commercial products consist not only of hardware such as an ED spectrometer and electronics but include powerful software packages as well. Additionally to managing the spectrum acquisition and analysis, the software can control the SEM using its external control interface. The utilization of a scan generator enables the acquisition of mappings or hyperspectral images (spectrum images). The Bruker Quantax EDS-system includes the software Esprit which additionally offers the option to set up batches of various tasks via a software interface, so that users can simply define a list of jobs, e.g. for certain acquisition types like a scan or map. This solves many analytical tasks already. But, a growing number of users need more complex types of acquisitions including the combination with data processing modules. The ability to interoperate and communicate with other software packages is another must have today.

One interface is not enough to provide that much flexibility. Therefore, various types of interfaces need to be integrated into the software. These, so-called application programming interfaces (APIs) can interact with the core software/hardware at different levels. In our terms the core software is Esprit and provided interfaces are (Fig. 1):

- a software API for certain types of acquisition or analysis methods

- $\quad$ an internal scripting API which can be used for batch processing tasks

- a data API for import and conversion of different types of data like e.g. spectra or mappings

- a hardware API to execute particular commands, enable a low-level control of the hardware and read raw data acquired by the detector

With the help of these interfaces a wide range of applications can be covered, and the user can apply the API without deeper knowledge of the internal software structure or specific EDS analysis routines. Thus, e.g. mining industry can quickly analyze and categorize minerals into classes using their highly specialized particle analysis tools. Regular monitoring and calibration of instrumentation can be automated [1]. The analysis of large data sets or their unattended transfer into an external e.g. 3Dsoftware package can be realized using an API. Finally, combined analyses of different methods can be performed by external software, such as EELS in combination with Quantax EDS at rates of one $\mathrm{kHz}$ [2].

For the design of the different APIs particular requirements are important. APIs should

- be independent of the programming language accessing them

- be as simple as possible

- offer flexible access to hard/software on different levels

- provide access to data structures without the need to know the internals

- provide data structures witch match each other wherever possible

The aim of this presentation is to give an overview of the different methods available to automate the work with the Bruker Esprit software. 


\section{References:}

[1] R. Wuhrer et al., Microsc. Microanal. 18 (Suppl. 2) (2012), 28

[2] P. Longo and R. Twesten, Microscopy Today 21 (2013), 28

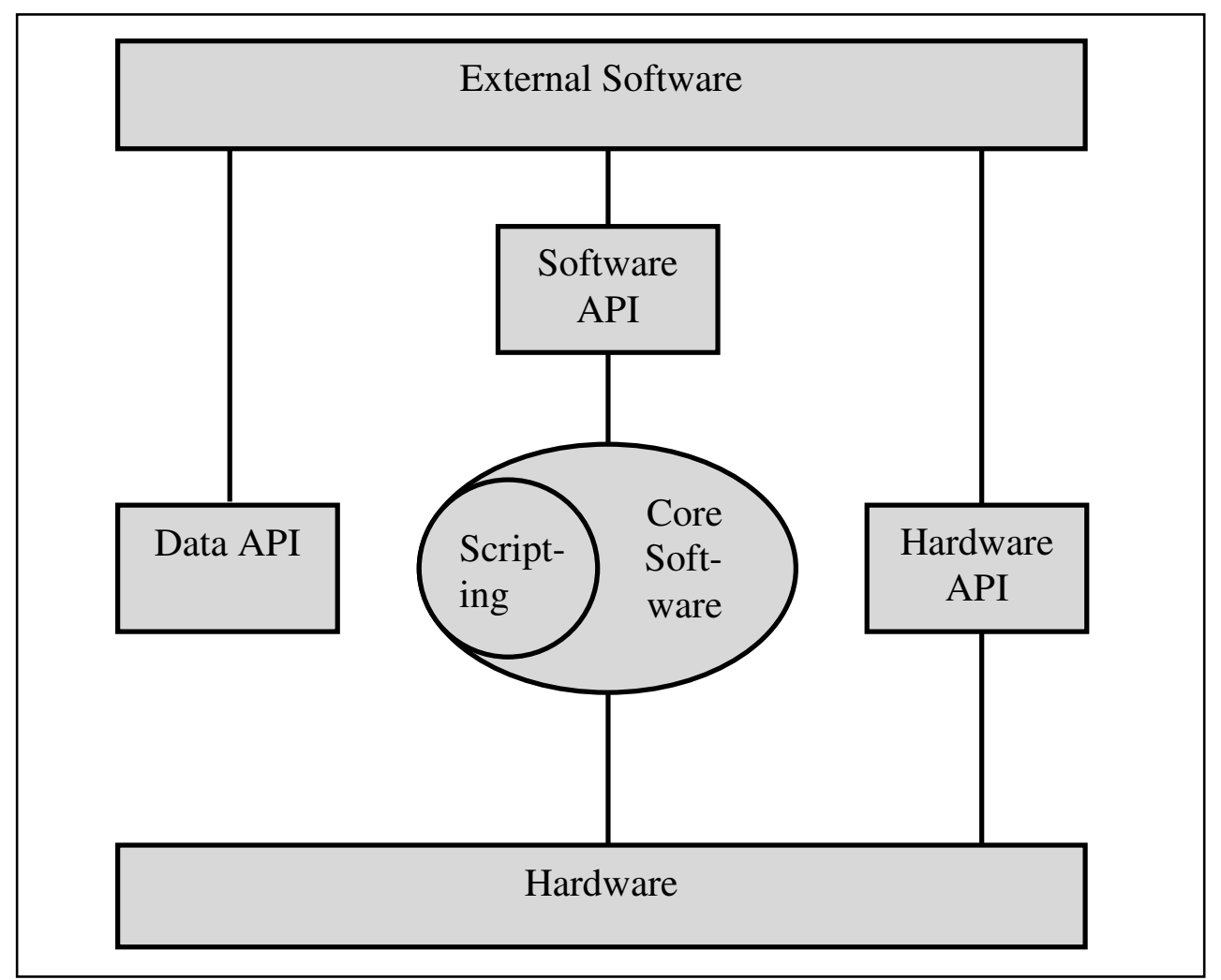

Fig. 1. Flow chart with the different APIs designed to access the software and hardware for various types of tasks. 